ASTIN COLLOQUIUM I966 ARNHEM 


\section{EXPERIENCE RATING AND CREDIBILITY}

\section{Hans Bühlmann \\ Zürich}

I. Examples of Experience Rating

Let me begin with some practical examples of experience rating.

a) Swiss Automobile Tariff 1963

- Within each tariff-position there are 22 grades:

\begin{tabular}{cc} 
Grade & $\begin{array}{c}\text { to pay } \% \text { of } \\
\text { basic premium }\end{array}$ \\
\hline $2 \mathrm{I}$ & 280 \\
20 & 280 \\
$\mathrm{I} 9$ & 280 \\
I8 & 200 \\
I7 & 200 \\
I6 & 200 \\
I5 & I40 \\
I4 & I40 \\
I3 & I40 \\
I2 & I00 \\
II & 100 \\
I0 & I00 \\
9 & I00 \\
8 & I00 \\
7 & I00 \\
6 & 80 \\
5 & 80 \\
4 & 80 \\
3 & 60 \\
2 & 60 \\
I & 60 \\
0 & 50
\end{tabular}


- The new owner of a car starts at grade 9

- The basic premium is determined on the basis of objective characteristics of the risk and essentially depends on the horsepower classification of the car

- The 22 grades are experience-rated as follows: For each accident one rises three grades and for each accident-free year one falls one grade. A driver who has I accident in every 4 years hence remains within four adjacent grades.

b) Sliding Scale Premiums in Reinsurance

Excess of Loss Contracts often stipulate that:

The rate of premium to be applied to the subject premium volume is determined at the end of the cover period as follows:

$$
\text { rate }=\frac{\text { Excess claims during period }}{\text { subject premium during period }}+\text { o,oI }
$$

subject to a minimum of 0,04

and a maximum of 0,08

\section{c) Participation in Mortality Profit in Group Life Insurance}

A group life insurance covers the members of the group on a one year term basis. It is often agreed that at the end of the year mortality profits are given back to the group according to the formula

$$
\text { refund }=x \% \text { gross premiums }-y \% \text { claims (where } x<y \text { ) }
$$

\section{The General Problem of Experience Rating}

All these examples fall under the heading "Experience Rating". What do they have in common?

Definition: A system by which the premium of the individual risk depends upon the claims experience of this same individual risk.

Note I: The emphasis is on claims experience of the individual risks as opposed to claims experience of a group of equal (or at least similar) risks, the latter being the viewpoint taken in "ordinary rating".

Note 2: There is no reason why an essential difference should be seen between the two cases 
I) where claims experience of previous periods only are entering into the experience rating formula (Swiss Automobile Tariff I963) or

2) where the claims experience of the period for which the premium applies is also taken into consideration (sliding scale premium, profit refund formula).

Both fall under the general heading Experience Rating.

The actuary confronted with such an experience rating problem is asked to establish a function assigning a premium (or rate) to every claims experience. Rating therefore which in classical actuarial techniques has always consisted of finding a fixed rate (i.e. a number) now becomes the problem of finding a suitable function.

\section{Description of the General Problem of Experience RATING *)}

Since we are asked to find "a suitable function" (when confronted with any experience rating problem), it seems natural to try to describe the "General Problem of Experience Rating" by means of the language of functional relationships:

domaine

$$
X=\left\{\left[x_{1}, x_{2}, \ldots x_{n}\right]\right\}
$$

all possible claims experiences in a (possibly unlimited) number of periods

$$
\text { range } \quad S=\{s\}
$$

all possible premiums (rates) which may be assigned to the individual risk

functional relationship

$$
\vec{R}=\left\{R_{1}, R_{2}, \ldots R_{n}\right\}
$$

where $R_{k}$ assigns a premium to the $k$-th period as a function of the values taken by $\left[x_{1} \ldots x_{k-1}\right]$ (or $\left.\left[x_{1} \ldots x_{k}\right]\right)\left(R_{k}\right.$ assigns an element of $S$ to every element of $X$ ).

*) A similar description is given in paper ( $\mathrm{r}$ ) (in German). 
In the example of the Swiss Automobile Tariff

$$
\begin{aligned}
X= & \text { all integer-valued non-negative sequences } \\
S= & \text { all possible grades from o to } 22 \\
R_{n}= & 9-(n-\mathrm{I})+\sum_{i=1}^{n-1}\left[3 x_{i}+\min \left(\mathrm{I}, x_{i}\right)\right] \\
& \text { but in any case } 0 \leqslant R_{n} \leqslant 22
\end{aligned}
$$

\section{Purpose of Experience Rating}

One might feel tempted to state that any Experience Rating Formula should aim at equating premiums to claims. The obvious conclusion to be drawn from this statement would therefore be that this is best achieved if everybody were to pay his own claims. This can certainly not be the idea of experience rating!

A more successful definition of the purpose of experience rating is obtained as follows:

- Experience Rating aims at assigning to each individual risk its

- own correct premium (rate)

- The correct premium for any period depends exclusively on the (unknown) claims distribution of the individual risk for this same period.

Using the symbols explained above let $F_{n}(x)$ be the probability distribution function of $x_{n}$ $s_{n} \quad$ the correct premium for the period $n$ e.g. $\left.s_{n}=\int x d F_{n}(x) s_{n}=\int x d F_{n}(x)+\alpha \sqrt{\int x^{2} d F_{n}(x)-\left[\int x d F_{n}(x)\right.}\right]^{2}$

The problem of experience rating can thus be formulated:

"Estimate for each period $n$ the correct premium $s_{n}$ based upon the observations made on $x_{1}, x_{2}, \ldots x_{n-1}$ (and possibly $x_{n}$ )"; in other words $R_{n}\left(x_{1} \ldots x_{n-1}\right)$ (or $R_{n}\left(x_{1} \ldots x_{n}\right)$-in our previous notation - is the estimator for the correct rate $s_{n}$.

$$
\begin{aligned}
& \text { Example: } x_{i} \sim P(\lambda+\gamma i) \text { independent } \\
& \lambda \text { unknown } \\
& \gamma \text { unknown } \\
& \text { correct premium } s_{n}=C \cdot E\left(x_{n}\right)=C \cdot\left[\lambda+\gamma_{n}\right] \\
& \text { Problem: Estimate } s_{n} \text { based upon observations } \\
& x_{1}, x_{2}, \ldots x_{n-1},\left(x_{n}\right) \text {. }
\end{aligned}
$$




\section{The Structure of the Portfolio}

So far we have been dealing with the individual risk alone, since we have looked at experience rating as the procedure which determines the correct rate of the individual risk from the experience of the very same risk.

It is most remarkable that, in spite of this individualistic view of experience rating, the techniques used for experience rating purposes only become meaningful if the structure of the portfolio containing the individual risk is also taken into account.

It is the old concept of the "collective of risks" which turns out to be of utmost importance also within the experience rating framework. But it is no longer the (purely fictitious) concept of a homogeneous collective. The collective, considered for experience rating purposes, may very well be heterogeneous.

\section{Example:}

- All automobile drivers within the same horsepower class

- All group life insurances written by a life insurance company

- All reinsurance contracts of the same form covering the same branches but different companies.

Whenever we are confronted with such a heterogeneous collective (portfolio) we shall use the following notation:

$\theta$ parameter characterizing the individual risk

$\Theta=\{\theta\}$ portfolio of risks $=$ collection of parameters characterizing the individual risks.

$s_{n}(\theta), F_{n}{ }^{(\theta)}(X), x_{n}{ }^{(\theta)}$ denote the correct rate, the distribution function of the $n$-th observation, the random variable standing for the $n$-th observation all apartaining to the individual risk $\theta$

where there is no possibility of confusion we shall very often omit $\theta$

For all portfolios under consideration, we assume that for each (measurable) subset $\Theta^{\prime}$ of $\Theta$ we know the percentage of risks contained therein.

The function $S\left(\Theta^{\prime}\right)$ which indicates this percentage is called the structural function. 
Example:

Risks are characterized by $\theta=$ expected total amount of claims caused by the risk during one period

then e.g.

$S\left(\Theta^{\prime}\right)=\frac{c}{\sqrt{2 \pi}} \int_{\Theta^{\prime}} e^{-\frac{\left(\theta-\mu_{0}\right)^{2}}{2}} d \theta$ structural function normal truncated

This structural function may be found by analysis of the portfolio

e.g. Swiss Automobile Tariff

$\theta=$ expected number of claims (independent of year of observation)

$$
\begin{gathered}
S\left(\Theta^{\prime}\right)=\frac{c \gamma}{\Gamma(\gamma)} \int_{\Theta^{\prime}} \theta^{\gamma-1} e^{-c \theta} d \theta \\
\gamma=\mathrm{I} \quad c=6.45
\end{gathered}
$$

However, in cases where such analysis is not possible or not available, what then? The structural function may of course still be assessed on the basis of "informed underwriting judgment" thereby expressing one's belief in what one thinks the structural function might look like. This may seem unscientific to you, since it clearly introduces a subjective element. In defence of such a procedure--which by the way is also the object of hot controversies among professional statisticians-I only wish to point out that it was in fact those theoreticians who have grown out of insurance, Arthur Bailey, Bruno de Finetti-just to mention two very prominent names-who were the earliest advocates of subjective assessments. Why? Because, if we want to be honest about our business we must admit that we have never been able to accomplish our duties without subjective judgments. Just consider for a moment old age mortality, the rating of unusual risks, the assessment of accumulation hazards etc. And this is the important fact: We have found - in cases of uncertainty-that our decisions are more successful, if we express our subjective feelings about this uncertainty rather than abstain from any judgment about the uncertain elements. 
6. How to Construct an Experience Rating Formula

We have now all the elements available to construct an Experience Rating Formula:

- The description of the Experience Rating Problem

- The structural function indicating the composition of the portfolio

Which properties do we expect from the Experience Rating Formula?

Sets of Postulates $A$

$$
\int_{\Theta} s_{n}(\theta) d S(\theta)=\int_{\Theta} E_{\theta}\left[R_{n} \overrightarrow{(x)}\right] d S(\theta)
$$

sum of correct premiums over the portfolio = expected income by experience rating formula from the portfolio.

$$
\int_{\theta} E_{\theta}\left[R_{n} \overrightarrow{(x)}-s_{n}(\theta)\right]^{2} d S(\theta)=\text { minimum }
$$

sum of square deviations from the correct individual premium as small as possible (limitation on fluctuations)

Sets of Postulates $B$

$$
\int_{x^{\prime} \times \Theta} s_{n}(\theta) d G_{n}^{(\theta)} \overrightarrow{(x)} d S(\theta)=\int_{x^{\prime} \times \Theta} R_{n}\left(\overrightarrow{x)} d G_{n}^{(\theta)} \overrightarrow{(x)} d S(\theta)\right.
$$

where $\quad G_{n}^{(\theta)}\left(\overrightarrow{x)} n\right.$-dimensional distribution over $x_{1}, x_{2} \ldots x_{n}$ $X^{\prime}$ subset of $X$

sum of correct premiums over any part of portfolio characterized by claims experience $=$ expected income by experience rating from the same part of the portfolio (impossibility of antiselection)

\section{Theorem:}

Postulates A and/or Postulates B lead to the same experience rating formula

$$
R_{n} \overrightarrow{(x)}=E\left[s_{n}(\theta) \overrightarrow{/ x}\right] \text { a posteriori expectation }
$$




\section{Specialization}

Up to this point I have developed the theory as I see it in its widest possible application. From here there are many roads to specialization. Permit me just to sketch a few possibilities and to indicate some rather personal conjectures for future research.

r) Homogeneity in time

$x_{k}^{(\theta)} k=\mathrm{I}, 2, \ldots n$ independent and identically distributed for fixed $\theta$

2) Credibility linear approximation to (I)

$$
a \int s_{n}(\theta) d S(\theta)+b \frac{x_{1}+x_{2}+\ldots x_{n}}{n} \sim E\left[s_{n}(\theta) \overrightarrow{/ x}\right]
$$

least square approximation

Conjecture:

This linear form is particularly meaningful in the case of a distribution function of the observations for which the average $\bar{x}$ is a sufficient statistic (normal, Poisson, Binomial). For cases where $\Sigma \log x_{i}$ is a sufficient statistic (log normal-, $\gamma$-distribution) it might be better to approximate through

$$
a \int s_{n}(\theta) d S(\theta)+b \frac{\sum \log x_{i}}{n}
$$

It would therefore appear that this latter formula might be more useful for experience-rating of claims amounts, whereas for experience-rating the frequency of claims the earlier formula would be more suitable.

\section{3) Heterogeneity in time (but still stochastic independence)}

This allows experience rating procedures only if, in spite of the heterogeneity, there remains some form of coherence between past and future. The classical case of coherence is that of trend-in particular the linear trend

e.g.

$$
\begin{aligned}
& x_{n} \sim P([n+k] \theta) k \text { fixed independent of } \theta \\
& s_{n}(\theta) \sim(n+k) \theta \\
& R_{n}=E\left[\theta / x_{1}, \ldots x_{n}\right]
\end{aligned}
$$


in particular

$$
R_{n}=[k+n)\left\{E[\theta] \frac{c}{c+\Sigma[i+k]}+\frac{\Sigma x_{i}}{\Sigma[i+k]} \cdot \frac{\Sigma[i+k]}{c+\Sigma[i+k]}\right\}
$$

in the case of a $\Gamma$-structural function. The credibility type formula thus appears even in the case of heterogeneity in time-though in a very special case.

This last specialization which tackles the heterogeneity in time appears to me to be very promising. As we live in a constantly changing world, we ought to adapt our models to this reality of change.

\section{ConCLUSion}

There was a very long delay before actuaries started to continue those thoughts which Ove Lundberg [2] and Arthur Bailey [3, 4] originated on both sides of the Atlantic about thirty years ago and as far as I know, independently of each other. The reason for this delay can be mainly seen in the objectivistic wave which has dominated all thinking within mathematical statistics during the last thirty years.

With the breakthrough of a more subjectivistic view of statistical problems, at least as an acceptable alternative, experience rating as regarded by these two early authors (and as treated here) has become a central part of all acturial activity.

\section{BIBLIOGRAPHY}

[I] Bühlmans, H.: “Optimale Prämienstufensysteme”, Mitteilungen Vereinigung schweizerischer Versicherungsmathematiker, 64. Band-Heft 2 (1964).

[2] Lundberg, O.: On Random Processes and their Application to Sickness and Accident Statistics (I940).

[3] BaIley, A.: “Credibility Procedures, Laplace's Generalization of Bayes' Rule and the Combination of Collateral Knowledge with Observed Data", Proceedings of the Casualty Actuarial Society, Vol. 37 (I950).

[4] BaIley, A.: "A Generalized Theory of Credibility", Proceedings of the Casualty Actuarial Society, Vol. 32 (I945). 